\title{
População indígena: uma reflexão sobre a influência da civilização urbana no estado nutricional e na saúde bucal
}

\author{
Indigenous peoples: reflection about the \\ influence of urban civilization on \\ nutritional status and oral health
}

Patrícia Garcia de MOURA'

Luciana Rodrigues Vieira BATISTA ${ }^{1}$

Emilia Addison Machado MOREIRA ${ }^{2}$

\section{RE S U M O}

Embora ainda existam sociedades indígenas que mantêm um modo tradicional de viver, nas quais uma alimentação adequada em quantidade e qualidade provém da caça e da prática de agricultura, a maioria dos índios vem sofrendo um processo de aculturação oriundo do contato com a civilização urbana, pois eles não mais dispõem de terra suficiente que garanta a subsistência. Desse modo, acabam tornando-se dependentes do comércio local e consumindo alimentos industrializados que podem ser prejudiciais à saúde bucal e ao estado nutricional. A presente comunicação tem como objetivo identificar a influência da transição alimentar, devido ao contato com a civilização urbana, no estado nutricional e na condição bucal da população indígena brasileira. Os dados relacionados nessa investigação foram obtidos na base de dados do SciELO e do MedLine, PubMed, pesquisando-se os termos de indexação: "índios sul-americanos", "comportamento alimentar", "estado nutricional", "saúde bucal". Estudos revelaram a prevalência de desnutrição infantil, sobrepeso e obesidade em adultos, índice de cárie elevado e doença periodontal nas comunidades indígenas que vêm passando por mudanças de hábitos culturais. Conclui-se que a transição alimentar pode levar a desordens nutricionais e ao desenvolvimento de doenças bucais, sugerindo a necessidade de uma intervenção e/ou monitoramento por meio de programas dirigidos à assistência à saúde, ao saneamento básico, ao acesso à terra e à educação.

Termos de indexação: Estado nutricional. Índios sul-americanos. Saúde bucal. Transição nutricional.

A B S T R A C T

Although there are still some indigenous societies that have maintained their traditional way of life, where food consumption in terms of amount and quality is based on hunting and agriculture, most indigenous

\footnotetext{
$\overline{1}$ Universidade Federal de Santa Catarina, Programa de Pós-Graduação em Odontologia. Florianópolis, SC, Brasil.

2 Universidade Federal de Santa Catarina, Centro de Ciências da Saúde, Programa de Pós-Graduação em Nutrição e Odontologia, Departamento de Nutrição. Campus Universitário, s/n., Trindade, 88040-970, Florianópolis, SC, Brasil. Correspondência para/Correspondence to: E.A.M. MOREIRA.E-mail: <addison@ccs.ufsc.br>.
} 
peoples have been undergoing an acculturation process secondary to the contact with urban civilizations, since they no longer have enough land to guarantee their subsistence. Thus, they become dependent on local trade and consume processed foods that may be harmful to their oral health and nutritional status. The objective of this communication is to identify the influence of the dietary transition due to contact with urban civilization on the nutritional status and oral condition of native Brazilian populations. The data included in this investigation were obtained from the SciELO and MedLine, PubMed databases using the keywords: "South American natives", "dietary behavior", "nutritional status", "oral health". Studies have revealed a prevalence of childhood malnutrition, adult overweight and obesity, high caries rates and periodontal disease in the native Brazilian communities that have been experiencing changes in their cultural habits. In conclusion, dietary transition can lead to nutritional disorders and the development of oral diseases, suggesting the need for health care and basic sanitation programs and access to land and education, in order to intervene in or monitor these occurrences.

Indexing terms: Nutritional status. South american indians. Oral health. Nutritional transition.

\section{N T R O D U Ç Ã O}

Embora ainda existam sociedades indígenas que mantêm um modo tradicional de viver, nas quais uma alimentação adequada em quantidade e qualidade provém da caça e da prática de agricultura ${ }^{1,2}$, a maioria dos índios no Brasil não dispõe de terra suficiente que garanta a subsistência, principalmente por questões políticas e econômicas internas, degradação do ecossistema - com pouca mata nativa, e muita terra arrendada à agroindústria ${ }^{3}$. Além do abandono às práticas tradicionais de cultivo, que no passado contribuíam para a diversidade dos alimentos consumidos, os povos indígenas estão expostos a transformações socioeconômicas, o que os coloca em situação de alta vulnerabilidade diante dos problemas de ordem alimentar, nutricional e de saúde bucal ${ }^{4-7}$.

Somado a isso, eles passaram a consumir alimentos industrializados, em especial o açúcar, o que influenciou na alteração do perfil de saúde bucal identificado na população indígena ${ }^{4,8}$. Além disso, mudanças no preparo dos alimentos, sendo consumidos cozidos, tornando-os moles e adesivos, teriam facilitado o acúmulo de placa bacteriana nos dentes, elevando o potencial cariogênico e contribuindo para evolução da doença periodontal ${ }^{4}$.

Diante de tal quadro, e por se tratar de populações vulneráveis em relação a suas condições de nutrição e saúde bucal, este trabalho teve como objetivo identificar a influência das transições alimentares e culturais na saúde bucal e no estado nutricional da população indígena brasileira por meio de uma revisão da literatura, realizada na base de dados do SciELO e do MedLine, PubMed. Os termos de indexação usados nos idiomas português e inglês foram respectivamente: índios sul-americanos - indians, South American; estado nutricional - nutritional status; saúde bucal - oral health. Dentre os artigos encontrados, foram selecionados aqueles com metodologia semelhante tanto para a análise do estado nutricional quanto para a avaliação da condição de saúde bucal, com número amostral significante, sem restrição quanto à faixa etária e que relatassem a existência de contato ou não com a civilização, indicando a ocorrência ou não de transição cultural e, consequentemente, alimentar.

\section{Efeitos da transição cultural-alimentar no estado nutricional}

A distribuição da população indígena em 1999, segundo o Instituto Brasileiro de Geografia e Estatística (IBGE), representava um total de 271967 índios na zona urbana e 90923 que ainda vivem na zona rural ${ }^{9}$.

Com a expansão europeia pelo continente americano, apareceram drásticas alterações nos perfis de saúde e nutrição das sociedades indígenas, principalmente devido às epidemias por doenças infectoparasitárias ${ }^{10}$. Os estudos longitudinais de uma mesma aldeia ou estudos comparativos realizados entre aldeias que vivem do 
modo tradicional têm mostrado uma prevalência de desordens nutricionais naquelas que passaram por transformações culturais ${ }^{11,12}$. A subnutrição aparece com maior incidência nessas populações, sendo a principal causa de óbitos infantis ${ }^{10,13}$.

Dessa forma, o consumo inadequado de alimentos, em termos qualitativos e quantitativos, pode ter contribuído para o desenvolvimento de subnutrição, diarreia, desidratação, anemia e aumento da mortalidade infantil, como mostra o estudo com os Mbyá de Bracuí, no município de Angra dos Reis $(R J)^{10}$. Acredita-se que a grande quantidade de óbitos por desnutrição na população Kaingáng, do Estado do Rio Grande do Sul, poderia estar associada ao arrendamento das terras para colonos, o que restringiu o espaço para plantio de subsistência ${ }^{14}$.

O contato com a civilização urbana levou essa população a consumir "comida do branco" - alimentos industrializados - e a uma redução da atividade física, pois o que antes era obtido pela caça, pesca e agricultura de subsistência - plantavam-se milho, mandioca, banana, amendoim, cana, batata-doce, abacaxi e coletava-se mel - passou a ser adquirido pelo trabalho remunerado nos centros urbanos ou na produção e venda de artesanatos ${ }^{10,15}$. Um exemplo dessa transição cultural foi observado nos Xavante de Mato Grosso, com a queda no consumo de alimentos cultivados e um aumento dos industrializados - açúcar, café, óleo de cozinha, farinha de trigo, sal, pão, biscoitos, refrescos em pó, refrigerantes e balas ${ }^{16}$.

Estudos de avaliação do estado nutricional de indígenas atribuíram seus achados tanto de desnutrição quanto de obesidade e sobrepeso à transição cultural e alimentar a que essas populações foram submetidas ${ }^{12,17-21}$. Na comunidade indígena Xavante, do Brasil Central, onde foram avaliados 549 índios, aproximadamente um terço das crianças apresentou médias de estatura e peso baixas para a idade, revelando um quadro de desnutrição na infância. Em relação aos adultos, 70\% apresentaram um aumento nas frequências de sobrepeso e obesidade, sendo encontrados altos valores para o Índice de Massa Corporal (IMC). Essa coexistência de formas distintas de má-nutrição aponta para questões ecológicas e socioeconômicas relevantes, em particular no que tange à sustentabilidade alimentar ${ }^{12,19}$.

Em análise comparativa do perfil ecológico-humano e antropológico das comunidades indígenas Xavante Etéñitépa e São José, do Estado de Mato Grosso, observou-se que a comunidade que desenvolve principalmente atividades ligadas à horticultura, à pesca e à caça exibiu valores do IMC inferiores aos de Etéñitépa, que apresentava prevalência superior de obesidade ${ }^{7}$.

Os indígenas Kaiowá, do Mato Grosso do Sul, que passaram por mudanças sociais, econômicas e ambientais decorrentes da perda da floresta como espaço vital, sofreram deterioração do estado nutricional com consequências na saúde ${ }^{17,18}$. O processo de envolvimento com a vida urbana, somado a outros fatores, contribuiu para a simultaneidade de problemas avaliados - déficits nutricionais e retardo de crescimento, caracterizando elevada prevalência de desnutrição infantil - que levam à atual precária condição de vida das 64 famílias da comunidade indígena Terena, do Mato Grosso do Sul22.

Assim, as mudanças alimentares e/ou a transição no padrão alimentar podem constituir um período crítico em relação aos riscos nutricionais ${ }^{23}$. Nos índios, são atribuídas ao processo de aculturação oriunda do contato com a civilização24.

O número reduzido de estudos com populações indígenas que ainda vivem de modo tradicional e afastadas da civilização urbana pode ser explicado pela própria dificuldade de acesso dos pesquisadores. Em contrapartida, a maioria dos estudos contemplou aldeias em transição cultural e alimentar e encontraram situações precárias de saúde, o que reforça a necessidade de incorporar programas que melhorem a assistência à saúde, o saneamento básico, a educação e o acesso às terras. 


\section{A condição bucal da população indígena}

Ainda são poucos os estudos relacionados à saúde bucal dessas populações. Povos de tribos isoladas na mata e submetidos a uma alimentação sem contato com o açúcar e com uma dieta baseada na caça e na coleta de frutos e raízes silvestres costumam apresentar prevalência mínima de cárie ${ }^{25}$. Já o contato com a civilização brasileira ocidentalizada e com seus hábitos alimentares tem produzido um efeito devastador sobre a dentição desses povos ${ }^{25,26}$.

Tal fato foi observado em um estudo epidemiológico ${ }^{4}$ que realizou um levantamento em saúde bucal em 228 indivíduos da comunidade indígena Xavante Etéiñitépa, em 1997, e que foi posteriormente comparado com os conduzidos em 1962 e 1991, revelando uma tendência de deterioração das condições de saúde bucal ao longo do tempo, ainda que se encontre em níveis considerados baixos, com índice de Dentes Cariados, Perdidos e Obturados na Dentição permanente (CPOD) igual a 2,6. Argumenta-se que mudanças na alimentação - notadamente consumo de açúcar e de outros produtos industrializados, relacionadas a alterações socioeconômicas e ambientais advindas da interação com a sociedade nacional envolvente, aliadas à falta de programas preventivos, são as principais causas da má condição bucal dessa população4 .

Os índios do Alto Xingu ( $n=288)$ apresentaram elevados índices de cáries em todos os grupos etários e uma proporção expressiva de dentes perdidos nos adultos ${ }^{27}$. Uma associação positiva entre a experiência de cárie e a exposição à cultura ocidental foi observada em índios Yanomami $(n=220)$ de três aldeias de diferentes graus de contato com a cultura não indígena, na fronteira do Brasil com Venezuela ${ }^{28 .}$

Em uma pesquisa qualitativa sobre práticas em saúde bucal entre os Guarani-Mbyá do Estado de São Paulo, o índio entrevistado relata que nos mais velhos "não dá dor de dente", mas nas crianças sim, pois, segundo o informante, "elas comem muito açúcar". Nesse mesmo estudo, outro infor- mante revela que, no tempo de seus avós, "os dentes eram bons", pois a alimentação era diferente ${ }^{29}$.

Estudo realizado entre os índios Guarani-Kaiowá, do Mato Grosso do Sul, menciona a associação entre o agravamento das condições de saúde bucal e o incremento do consumo de alimentos industrializados de alto potencial cariogênico ${ }^{8}$, concordando com Arantes et al. ${ }^{4}$, que atribui a má condição bucal dos Xavantes ao notável consumo de açúcar. Nos índios Xavante foi observada baixa ocorrência de doença periodontal em estágios avançados, apesar de não haver uma forma de higienização oral sistemática e difundida entre eles, podendo-se atribuir o fato à resistência a fatores irritativos locais ou à alimentação constituída de alimentos duros e fibrosos ${ }^{4}$

Em uma revisão sobre aspectos gerais da obesidade e da cárie dentária, os autores, embasados em 32 referências, enfocaram a transição alimentar como problema comum ao desenvolvimento de $\mathrm{ambas}^{30}$. Assim como a nutrição e os hábitos alimentares têm grande influência sobre a saúde dentária, visto que hábitos saudáveis de alimentação asseguram uma boa saúde geral e bucal $^{31}$, manter uma dentição natural funcional desempenha um importante papel em ter uma dieta saudável e um estado nutricional satisfatório ${ }^{32}$.

Antigamente, era comum utilizarem os "remédios do mato", feitos pelo pajé em forma de chá, para curar dores de estômago e de dente, inflamações em geral, tranquilizantes e até mesmo como anticoncepcionais ${ }^{10}$. Hoje em dia, a má condição de saúde e os números elevados de óbitos por causas evitáveis na população indígena, consequentes das mudanças no seu modo de vida, implicam a necessidade de utilizarem os serviços do então criado Subsistema de Saúde Indígena, articulado com o Sistema Único de Saúde, para atender às especificidades desses povos ${ }^{14,33}$.

Em termos genéricos, a população indígena está, pelas próprias condições de vida atual, mais exposta à inadequação alimentar e, consequentemente, a problemas bucais. 


\section{CONSIDERAÇÕES FINAIS}

Os povos indígenas no Brasil vêm experimentando, ao longo do tempo, experiências distintas de interação com a sociedade nacional. Não obstante, é inquestionável a intensidade dos processos de mudanças socioeconômicas, culturais e ambientais, com amplos impactos sobre a saúde.

O acesso limitado a serviços de educação e saúde, os conflitos por terras e a absorção do indígena nos mercados regionais têm favorecido a migração indígena para as cidades brasileiras ${ }^{34}$. Isso acarreta um processo de aculturação que conduz a uma transição alimentar, visto que a população passa de uma atividade de subsistência baseada na caça, pesca e coleta para uma incorporação de alimentos industrializados, desviando seus esforços produtivos para culturas comerciais, para arrecadar dinheiro a fim de satisfazer às necessidades criadas com o novo hábito sociocultural. É importante lembrar a importância dos modos tradicionais de aquisição de alimentos na cultura indígena, que significa manter o corpo, a alma e a natureza em contato. Nesse sentido, a garantia da terra, tantas vezes reivindicada pelas lideranças, outras ações transdisciplinares e a participação comunitária devem ser priorizadas para promoção da segurança alimentar e nutricional ${ }^{6}$. As mudanças culturais e no padrão alimentar não acompanham o nível social e econômico em que essas populações vivem. Dessa forma, tais populações acabam sofrendo consequências no estado de saúde, como desordens nutricionais e desenvolvimento de doenças bucais (Quadro 1).

Apesar do aumento da produção científica sobre o tema e da melhora na metodologia dessa produção, ainda há acentuadas lacunas no que diz respeito à qualidade dos dados apresentados. A ampla maioria dos estudos é de prevalência. Em muitos estudos não foi possível identificar os procedimentos amostrais utilizados, tendo em geral prevalecido a amostragem por conveniência. Uma importante parcela dos estudos não apresenta os resultados, especificando a ocorrência da doença cárie por gênero e/ou idade. Há heterogeneidade quanto aos critérios de diagnóstico adotados em relação à avaliação nutricional. Já para a determinação das condições de saúde bucal, os estudos têm utilizado o índice CPOD e ceod (cariados, com extração indicada e obturados na dentição decídua). Há pouca uniformidade quanto à apresentação dos fatores de exposição, tornando difícil o resgate dessas informações. Em geral, os estudos apontam para altas prevalências de problemas bucais, bem como de inadequação nutricional. É também apontada a necessidade de uma maior padronização das pesquisas sobre saúde bucal e

Quadro 1.Comparação entre comunidades indígenas brasileiras que mantêm o modo tradicional de viver e comunidades que sofreram transições culturais, ambientais e alimentares.

\begin{tabular}{|c|c|}
\hline $\begin{array}{l}\text { Comunidades que ainda mantêm o modo tradicional de } \\
\text { viver (Yanomami, Médio Rio Negro'; Ikpeng, Alto Xingu² e } \\
\text { Xavante Etéñitepá, Mato Grosso }{ }^{7} \text { ) }\end{array}$ & $\begin{array}{l}\text { Comunidades que sofreram mudanças culturais e ambientais (Terena, } \\
\text { Mato Grosso do Sul }{ }^{6} \text {; Xavante São José, Mato Grosso7; Kaiowá, Ma- } \\
\text { to Grosso do Sul8; Guarani-Mbyá, São Paulo }{ }^{10,30} \text { e Xavante Sangra- } \\
\text { douro, Mato Grosso }{ }^{12} \text { ) }\end{array}$ \\
\hline Alimentação obtida pela caça/pesca e coleta (subsistência) & $\begin{array}{l}\text { Alimentos são comprados com dinheiro de trabalhos remunerados } \\
\text { nas cidades (principalmente vendas de artesanatos) ou recebem-se } \\
\text { doações de cestas básicas }\end{array}$ \\
\hline Alimentos duros e fibrosos, frutas, verduras e legumes & $\begin{array}{l}\text { Alimentos industrializados (enlatados, biscoitos, açúcar, refrigeran- } \\
\text { tes) }\end{array}$ \\
\hline Realizam atividade física intensa na obtenção dos alimentos & Reduzida atividade física \\
\hline $\begin{array}{l}\text { Valores normais ou menores de massa corporal e índice de } \\
\text { massa corporal }\end{array}$ & Prevalência de desnutrição infantil e de obesidade em adultos \\
\hline $\begin{array}{l}\text { Baixo índice de cárie e de ocorrência de doença periodontal } \\
\text { avançada }\end{array}$ & $\begin{array}{l}\text { Elevado índice de cárie nas crianças e elevada proporção de dentes } \\
\text { perdidos em adultos }\end{array}$ \\
\hline
\end{tabular}


estado nutricional de modo a aumentar a comparabilidade e, eventualmente, subsidiar os serviços de saúde com informações relevantes para efetuar intervenções.

\section{O N CLUS Ã O}

Nesse contexto, pode-se concluir que as acentuadas e rápidas mudanças culturais e ambientais vividas pelas diversas e diferentes comunidades indígenas influenciam o estado nutricional e a condição bucal. Além disso, mostra-se necessária uma intervenção ou monitoramento por meio de programas dirigidos à assistência à saúde, saneamento básico, acesso à terra e à educação, assim como a realização de estudos longitudinais que possam acompanhar as condições bucais e nutricionais desses indivíduos e, concomitantemente, atuar na promoção da saúde para comunidades indígenas que passam pelo processo de transição alimentar.

\section{A GRADECIMENTO}

A Lee Y Ching pela contribuição inicial na pesquisa bibliográfica em base de dados.

\section{COLABORADORES}

P.G. MOURA contribuiu com a idealização do artigo, fez o levantamento bibliográfico e redigiu o artigo. L.R.V. BATISTA contribuiu com a redação do artigo. E.A.M. MOREIRA contribuiu com a análise crítica e com a elaboração final da redação.

\section{REFERÊ NCIAS}

1. Istria J, Gazin P. O estado nutricional de crianças Yanomami do Médio Rio Negro, Amazônia. Rev Soc Bras Med Trop. 2002; 35(3):233-6. doi: 10.15 90/S0037-86822002000300006.

2. Fagundes-Neto U, Baruzzi RG, Oliva CAG, Kopelman B, Fagundes U. Avaliação do estado nutricional e da composição corporal das crianças índias do Alto Xingu e da etnia Ikpeng. J Pediatr.
2004; 80(6):483-9. doi: 10.1590/S0021-75572 004000800010.

3. Diehl E. Agravos na saúde Kaingáng (Terra Indígena Xapecó, Santa (atarina) e a estrutura dos serviços de atenção biomédica. Cad Saúde Pública. 2001; 17(2):439-45. doi: 10.1590/S0102-311X200100 0200019 .

4. Arantes R, Santos RV, Coimbra Jr CEA. A saúde bucal na população indígena Xavante de Pimentel Barbosa, Mato Grosso, Brasil. Cad Saúde Pública. 2001; 17(2):375-84. doi: 10.1590/S0102-311X20 01000200012 .

5. Coimbra Jr CEA, Santos RV. Avaliação do estado nutricional num contexto de mudança sócio-econômica: o grupo indígena Suruí do estado de Rondônia, Brasil. Cad Saúde Pública. 1991; 7(4): 538-62. doi: 10.1590/S0102-311X1991000400 006.

6. Fávaro T, Ribas DLB, Zorzatto JR, Segall-Corrêa AM, Panigassi G. Segurança alimentar em famílias indígenas Teréna, Mato Grosso do Sul, Brasil. Cad Saúde Pública. 2007; 23(4):785-93. doi: 10.1590/S0 102-311X2007000400006.

7. Gugelmin SA, Santos RV. Ecologia humana e antropometria nutricional de adultos Xavante, Mato Grosso, Brasil. Cad Saúde Pública. 2001; 17(2):313-22. doi: 10.1590/S0102-311X20010 00200006.

8. Parizotto SPCOL. Prevalência de cárie dentária na dentição decídua de crianças da comunidade indígena Kaiowá-Guarani de Mato Grosso do Sul e associação com fatores de risco [tese]. São Paulo: Faculdade de Odontologia da USP; 2004.

9. Instituto Brasileiro de Geografia e Estatística. Rio de Janeiro; 1999 [acesso 2007 jan 15]. Disponível em: <http://www.ibge.gov.br/ibgeteen/datas/indio/ numeros.html>.

10. Litaiff A. As divinas palavras: identidade étnica dos Guarani-Mbyá. Florianópolis: UFSC; 1996.

11. Martins SJ, Menezes RC. Evolução do estado nutricional de menores de 5 anos em aldeias indígenas da Tribo Parakanã, na Amazônia Oriental Brasileira (1989-1991). Rev Saúde Pública. 1994; 28(1):1-8. doi: 10.1590/S0034-89101994000100001.

12. Gugelmin SA, Santos RV. Uso do índice de massa corporal na avaliação do estado nutricional de adultos indígenas Xavante, Terra Indígena Sangradouro-Volta Grande, Mato Grosso, Brasil. Cad Saúde Pública. 2006; 22(9):1865-72. doi: 10.1590/ S0102-311X2006000900017.

13. Santos RV. Crescimento físico e estado nutricional de populações indígenas brasileiras. Cad Saúde Pública. 1993; 9(Suppl1):46-57. doi: 10.1590/S0 102-311X1993000500006. 
14. Hökerberg YHM, Duchiade MP, Barcellos C. Organização e qualidade da assistência à saúde dos índios Kaingáng do Rio Grande do Sul, Brasil. Cad Saúde Pública. 2001; 17(2):261-72. doi: 10.1590/S0102-311X2001000200002.

15. Litaiff A, Darella MDP. Os índios guarani mbyá e o parque estadual da Serra do Tabuleiro. Anais da $22^{a}$ Reunião Brasileira de Antropologia do Fórum de Pesquisa 3: conflitos socioambientais e unidades de conservação; 2000. Brasília; 2000.

16. Santos RV, Flowers NM, Coimbra Jr CEA, Gugelmin SA. Tapirs, tractors and tapes: the changing economy and ecology of the Xavante Indians of central Brazil. Hum Ecol. 1997; 25(4):545-66.

17. Brand A. Desenvolvimento local em comunidades indígenas no Mato Grosso do Sul: a construção de alternativas. Interações. 2001; 1(2):59-68.

18. Grünberg FP. Reflexões sobre a situação dos Guarani no Mato Grosso do Sul, Brasil. Brasília: Centro de Trabalho Indigenista; 2002.

19. Leite MS, Santos RV, Gugelmin SA, Coimbra Jr CEA. Crescimento físico e perfil nutricional da população indígena Xavante de Sangradouro-Volta Grande, Mato Grosso, Brasil. Cad Saúde Pública. 2006; 22(2):265-76. doi: 10.1590/S0102-311X20060 00200004.

20. Morais MB, Alves MS, Fagundes-Neto U. Estado nutricional de crianças índias terenas: evolução do peso e estatura e prevalência atual de anemia. J Pediatr. 2005; 81(5) 383-9. doi: 10.1590/S0021-7 5572005000600008.

21. Pícoli RP, Carandina L, Ribas DLB. Saúde materno-infantil e nutrição de crianças Kaiowá e Guarani, Área Indígena de Caarapó, Mato Grosso do Sul, Brasil. Cad Saúde Pública. 2006; 22(1):223-7. doi: 10.1590/S0102-311X2006000100025.

22. Ribas DLB, Sganzerla A, Zorzatto JR, Philippi ST. Nutrição e saúde infantil em uma comunidade indígena Terena, Mato Grosso do Sul, Brasil. Cad Saúde Pública. 2001; 17(2):323-31. doi: 10.1590/S0 102-311X2001000200007.

23. Brasil. Ministério da Saúde. Política nacional de alimentação e nutrição. $2^{a}$ ed. Brasília: Ministério da Saúde; 2003.

24. Schaden E. Aspectos fundamentais da cultura guarani. $3^{a}$ ed. São Paulo: EDUSP; 1974.
25. Pinto VG. Saúde bucal coletiva. $4^{\text {a }}$ ed. São Paulo: Santos; 2000.

26. Neel JV, Salzano FM, Junqueira PC, Keiter F, MayburyLewis D. Studies on the Xavante Indians of the Brazilian Mato Grosso. Am J Hum Genet. 1964; 16:52-140.

27. Rigonatto DDL, Antunes JLF, Frazão P. Dental caries experience in indians of the Upper Xingu, Brazil. Rev Inst Med Trop S Paulo. 2001; 43(2):93-8. doi: 10.1590/S0036-46652001000200008.

28. Donnelly CJ, Thomson LA, Stiles HM, Brewer C, Neel JV, Brunelle JA. Plaques, caries, periodontal diseases and acculturation among Yanomami Indians, Venezuela. Comm Dent Oral Epidem. 1977; 5(1):30-9. doi: 10.1111/j.1600-0528.19 77.tb01614.x

29. Oliveira MA. Representações e práticas em saúde bucal entre os Guarani Mbyá da aldeia Boa Vista no município de Ubatuba, São Paulo [dissertação]. São Paulo: Secretaria de Estado da Saúde de São Paulo; 2006.

30. Traebert J, Moreira EAM, Bosco VL, Almeida ICS. Transição alimentar: problema comum à obesidade e à cárie dentária. Rev Nutr. 2004; 17(2): 247-53. doi: 10.1590/S1415-52732004000200011.

31. Bönecker M, Sheiham A. Promovendo saúde bucal na infância e adolescência: conhecimentos e práticas. São Paulo: Santos; 2004.

32. Marcenes W, Steele JG, Sheiham A, Walls AWG. The relationship between dental status, food selection, nutrient intake, nutritional status, and body mass index in older people. Cad Saúde Pública. 2003; 19(3):809-16. doi: 10.1590/S0102$311 \times 2003000300013$.

33. Brasil. Fundação Nacional da Saúde. Atenção à saúde indígena. In: BRASIL, editor. 100 anos de saúde pública: a visão da FUNASA. Brasília; 2006.

34. Coimbra Jr CEA, Santos RV. Saúde, minorias e desigualdades: algumas teias de inter-relações, com ênfase nos povos indígenas no Brasil. Ciênc Saúde Coletiva. 2000; 5(1):125-32. doi: 10.1590/S14 13-81232000000100011.

Recebido em: 18/12/2007

Versão final reapresentada em: 11/12/2009 Aprovado em: 16/3/2010 